\title{
Comparative proteomic analysis of the function and network mechanisms of MASPIN in human lung cells
}

\author{
YAO LIU ${ }^{1}$, YI GENG $^{1}$, KUANZHI LI $^{1}$, FANG WANG $^{1}$, HAIPING ZHOU $^{1}$, \\ WANHU WANG ${ }^{1}$, JIE HOU ${ }^{1}$ and WENCHAO LIU ${ }^{2}$ \\ ${ }^{1}$ Baoji Center Hospital of Shanxi Province, Baoji, Shanxi 721008; ${ }^{2}$ Department of Clinical Oncology, \\ State Key Discipline of Cell Biology, Xijing Hospital, The Fourth Military \\ Medical University, Xi'an, Shanxi 710032, P.R. China
}

Received August 6, 2011; Accepted October 14, 2011

DOI: $10.3892 / \mathrm{etm} .2011 .427$

\begin{abstract}
MASPIN, which is also known as Serpin B5, is a novel tumor suppressor. Emerging evidence suggests that MASPIN acts as a multifaceted protein in various types of cancer, including prostate, breast and pancreatic cancer. It interacts with diverse groups of intercellular and extracellular proteins, regulating cell adhesion, motility, apoptosis and angiogenesis, and is involved in mammary gland development. As MASPIN is a multifunctional factor in cancer pathways, its function remains poorly illuminated. In this study, we compared the protein profiles of LC5 cell lines with MASPIN overexpression and knockdown using comparative two-dimensional gel electrophoresis. The differences in protein expression, visualized as differences in spots, were identified by time-of-flight (TOF)/TOF mass spectometry. Significant differences were observed between overexpressing and knocked down cells, including eight spots that were unique and sixteen spots that were up- or down-regulated by more than 4 -fold. Six genes, including Sdccag8, Ldoc1, SCAI, SDCCAG3, CT62 and NEDD9 were unique in MASPIN-expressing cell lines, but absent in knock-out cell lines, in which most of them play a significant role in the invasion of cancer cells. Moreover, the Brms1 and CAGE1 genes were identified as being uniquely expressed in knocked down cell lines, which were associated with the development and progression of tumors. The data from this study shed some light on the function, as well as the general network mechanisms of MASPIN in lung cancer.
\end{abstract}

\section{Introduction}

MASPIN, a novel serine protease inhibitor (Serpin), inhibits tumor invasion and metastasis of mammary carcinoma. The

Correspondence to: Dr Wenchao Liu, Department of Clinical Oncology, State Key Discipline of Cell Biology, Xijing Hospital, The Fourth Military Medical University, Xi'an, Shanxi 710032, P.R. China

E-mail: liuch@fmmu.edu.cn

Key words: MASPIN, lung cancer, two-dimensional gel electrophoresis gene encoding MASPIN has been initially identified in a search of genes that display no or down-regulated expression in human breast carcinoma cells (1). This $42-\mathrm{kDa}$ protein is known also as Serpin B5, protease inhibitor-5 or proteinase inhibitor-5. As a tumor suppressor gene, MASPIN is involved in tumor invasion, metastasis and angiogenesis. However, the mechanisms of MASPIN are little known, particularly its signal networks in lung cells.

MASPIN acts as a tumor suppressor gene by impairing tumor growth, cell motility and metastasis. Shi et al demonstrated that MASPIN reduced tumor growth as well as invasion and metastasis through a combination of reduced angiogenesis and increased apoptosis in a transgenic mouse model that overexpressed MASPIN (2). Abraham et al reported that MASPIN increased cell adhesion to the extracellular matrix in prostate tumor cells and that it was capable of decreasing the tumorigenic and metastatic potential of prostate tumors (3). The decreased expression of MASPIN in prostate cancer has also been reported to inversely correlate with the development of local recurrence or systemic tumor progression (4). Furthermore, it has been reported that MASPIN is involved in the processes of embryonic development, apoptosis and angiogenesis. Schaefer and Zhang demonstrated that MASPIN played a significant role in mammary development in a stage-dependent manner (5). The expression levels of MASPIN are lower in virgin and early pregnancy mammary glands than in late pregnancy and lactating mammary glands. Cher et al demonstrated that MASPIN expression inhibited osteolysis, tumor growth and angiogenesis in a model of prostate cancer bone metastasis (6). Finally, the local delivery of MASPIN to human prostate tumor cells in a mouse model blocked tumor growth and dramatically reduced the density of tumor-associated microvessels (5).

Over the past decade, with the expansion of studies on MASPIN, novel protein-binding partners have been identified and have provided insight into the molecular aspects on regulation and divergent mechanisms. Naturally, the MASPIN gene contains a number of potential transcription factor binding elements, which are related to the regulation of its expression. These include activator protein-1 (Ap-1), E26 transformation specific-1 (Ets), hormonal responsive elements (HREs) and p53 binding sites (5). Recently, it has been shown that 
MASPIN may be affected by promoter methylation levels and by histone modification (7). These data indicate the complexity of the MASPIN network. In this study, we used comparative proteomics to systemically study the protein profile changes in lung cell lines. The large-scale data and system biology methodology may help us to illustrate the function of MASPIN, its regulation pathways and networks in lung cancer.

\section{Materials and methods}

Cell culture. The human lung carcinoma cell line, LC5, was kindly provided by the Cell Line Center of the Chinese Academy of Science. The LC5 human lung carcinoma cell line was maintained in RPMI-1640 (Life Technologies, Gaithersburg, MD, USA) with $1 \mathrm{mM}$ glutamate, $100 \mathrm{U} / \mathrm{ml}$ penicillin, $100 \mathrm{ng} / \mathrm{ml}$ streptomycin and $10 \%$ fetal calf serum (FCS). The cells were cultured at $37^{\circ} \mathrm{C}$ in a humidified atmosphere of $5 \% \mathrm{CO}_{2}$. Cell viability was evaluated by trypan blue exclusion assay.

Porcine cytomegalovirus (pCMV)-maspin construction. For construction of the pCMV-MASPIN plasmid, full-length MASPIN complementary DNA (cDNA) was cloned into the pCMV-Taq4C vector (Invitrogen, Carlsbad, CA, USA), using the primers, 5'-atggatgccetgcaactagca-3' and 5'-ttaaggagaac agaatttg-3' (Sangon Co., Shanghai, China).

Transfection of the lung cancer cells. The cells were transfected with small interfering RNA (siRNA) or plasmids using the Effectene (Qiagen, Valencia, CA, USA) or Amaxa electroporation system (Amaxa, Gaithersburg, MD , USA), according to the manufacturer's instructions. To develop cell lines, which stably expressed MASPIN, following transfection, the cells were then incubated in the medium with Geneticin 418 for $48 \mathrm{~h}$, and selected for 2 weeks. We used a targeted SMART pool of siRNAs from Dharmacon (Lafayette, CO, USA) to knockdown MASPIN expression with the siLentFect lipid kit. In parallel, cells were transfected with a fluorescein isothiocyanate-labeled, nonspecific siRNA with a scrambled sequence.

Western blot analysis. Cells were harvested with ice-cold phosphate-buffered saline and then lysed in lysis buffer containing $10 \mathrm{mM}$ Tris ( $\mathrm{pH} 7.5), 150 \mathrm{mM} \mathrm{NaCl}, 10 \mathrm{mM}$ ethylenediaminetetraacetic acid, $1 \%$ SDS, $1 \mathrm{mM}$ sodium orthovanadate and a mixture of protease inhibitors (Roche, Indianapolis, IN, USA). The lysates were sonicated for 10 sec, centrifuged for $20 \mathrm{~min}$ at $20,000 \mathrm{x} \mathrm{g}$, and then stored at $-70^{\circ} \mathrm{C}$. Equal amounts $(25 \mu \mathrm{g})$ of the cell lysates were resolved by $12 \%$ sodium dodecyl sulfate polyacrylamide gel electrophoresis (SDS-PAGE) and were subjected to Western blot analysis, using an enhanced chemiluminescence system (Amersham Corp., Arlington Heights, IL, USA).

Two-dimensional gel electrophoresis (2DE) and time-of-flight (TOF)/TOF mass spectometry (MS). Proteins were collected from LC5 cells as described previously. The extraction buffer included: $9 \mathrm{M}$ urea, 0.5\% SDS, 4\% CHAPS, $65 \mathrm{mM}$ DTT, nucleases mixture (GE Healthcare, Piscataway, NJ, USA) and protease inhibitor mixture (GE Healthcare). The crude extraction was then treated with Clean-Up Kit (GE Healthcare). The protein $(100 \mu \mathrm{g})$ was loaded independently using $18 \mathrm{~cm} \mathrm{pH} 3-10$ (L) stripes by the GE Healthcare 2DE system. Second dimensional SDS-PAGE was carried out, using a $12 \%$ gel and stained with the PlusOne Silver Staining Kit (GE Healthcare). The gel images were analyzed by the ImageMaster ${ }^{\mathrm{TM}} 2 \mathrm{D}$ Platinum v7.0 (GE Healthcare). The spots were identified by 6200 Series Accurate-Mass TOF LC/MS (Agilent, Santa Clara, CA, USA).

\section{Results}

Overexpression and knockdown of MASPIN in LC5 human lung carcinoma cells. Total RNA was isolated from four exponentially selected cell lines with overexpressed and knocked down MASPIN, respectively. The results suggest that all eight cell lines were successful. All of the transfected cells had a high expression of MASPIN, while MASPIN expression was almost eliminated by siRNA-MASPIN (Fig. 1). Furthermore, the expression of MASPIN protein was confirmed by Western blot analysis. The abundance of MASPIN was detected in the overexpressed cell lines by a specific anti-MASPIN antibody, but was undetectable in the knocked down cell line (Fig. 2). The results indicated that the overexpression or knockdown of MASPIN in LC5 cell lines was successful.

Spot identification by TOF/TOF MS. The global view of a representative two-dimensional gel is shown in Fig. 3. The MASPIN overexpression and knockdown samples were loaded three times. Over 1,400 spots were represented in three duplicates. The excellent reproduction in protein pattern and protein density between all cell lines is evident in the comparison of four 2D gels to each other. All gels were matched to the LC5 cells reference 2D map and the relative density of each protein spot was quantified using ImageMaster software. The data are shown in Table I.

The results indicate that several tumor suppressor genes are related to the expression of MASPIN. In this study, we have found that at least six genes were unique in MASPIN-overexpressed cell lines, in which most of them play crucial roles in the invasion of cancer cells. For example, the suppressor of cancer cell invasion (SCAI) acts as an inhibitor of cancer cell invasion through the transcriptional control of $\beta 1$-integrin (8). The serologically defined colon cancer antigen 3 (SDCCAG3) is necessary for the presentation of TNF receptor 1 on the cell surface (9). Leucine zipper, down-regulated in cancer 1 (LDOC1) inhibits NF- $\mathrm{NB}$ activation and sensitizes pancreatic cancer cells to apoptosis (10). In addition, breast cancer metastasis suppressor 1 (Brms1) functions as a co-repressor by enhancing histone deacetylase 1-mediated deacetylation of RelA/p65 and promoting apoptosis, and inhibits gene expression by targeting NF- $\kappa$ B activity (11).

However, two genes were discovered to be unique in knockdown cell lines that are associated with the development and progression of tumors. The expression of cancer-associated gene 1 (CAGE1) protein is associated with the progression of tumors and has been used as a response criteria in several tumors during chemotherapy (12). The neural precursor cell expressed developmentally down-regulated protein 9 (NEDD9), as a melanoma metastasis gene, is regulated in human neuroblastoma cells and in the embryonic hindbrain by all-trans retinoic acid $(13,14)$. 
Table I. Spots identification by TOF/TOF MS.

\begin{tabular}{lccc}
\hline $\begin{array}{l}\text { Protein } \\
\text { name }\end{array}$ & $\begin{array}{c}\text { Quantity in MASPIN } \\
\text { overexpression sample (V \%) }\end{array}$ & $\begin{array}{c}\text { Quantity in MASPIN } \\
\text { knockdown sample (V \%) }\end{array}$ & $\begin{array}{c}\text { Ratio overexpression/ } \\
\text { knockdown }\end{array}$ \\
\hline Sdccag8 & Unique & - & - \\
Ldoc1 & Unique & - & - \\
SCAI & Unique & - & - \\
SDCCAG3 & Unique & - & - \\
CT62 & Unique & - & - \\
Brms1 & Unique & - & - \\
CAGE1 & - & Unique & - \\
NEDD9 & - & Unique & 24.0 \\
TSP1 & 0.288 & 0.012 & 17.3 \\
RUNX & 0.294 & 0.017 & 15.2 \\
ORAOV1 & 0.335 & 0.022 & 16.5 \\
PTK2 & 0.396 & 0.024 & 6.38 \\
CASC5 & 0.415 & 0.065 & 6.23 \\
BCAR1 & 0.088 & 7.04 \\
GREB1L & 0.583 & 0.096 & 4.54 \\
BASE & 0.676 & 0.152 & 4.18 \\
CTAG2 & 0.688 & 0.169 & 0.09 \\
Blcap, & 0.707 & 0.835 & 0.12 \\
Casc5 & 0.077 & 0.799 & 0.13 \\
Casc3 & 0.098 & 0.761 & 0.15 \\
Hic2 & 0.104 & 0.733 & 0.18 \\
SNCG & 0.112 & 0.704 & 0.21 \\
HIC1 & 0.127 & 0.698 & 0.23 \\
DLC1 & 0.144 & 0.668 & \\
\hline
\end{tabular}

TOF MS, time-of-flight mass spectrometry.

\section{Overexpression Knockdown}
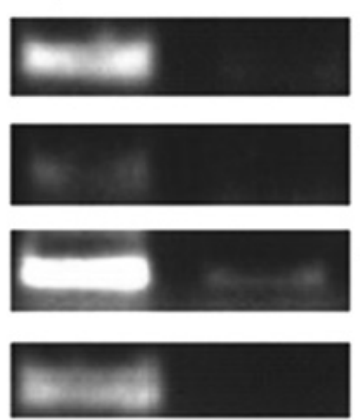

Transfected LC5 line 4

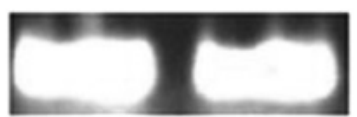

Actin

Figure 1. Northern (RNA) blot analysis of MASPIN mRNA in four overexpressed and knock-out cell lines.

Other findings from this study were that nine genes were extremely highly expressed in the MASPIN-overexpressed cell lines. Most of these highly expressed genes are associated with anti-thrombosis, inhibition of tumor cell mobility, metastasis and angiogenesis. For example, soluble or local platelet-released

\section{Knockdown Overexpression}

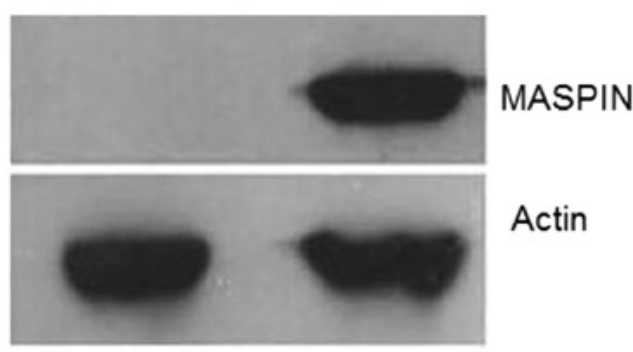

Figure 2. Western blot analysis of MASPIN protein in overexpressed and knock-out cell lines.

thrombospondin-1 (TSP-1) may protect unfolded endothelium-bound and subendothelial von Willebrand factor (VWF) from degradation by plasma ADAMTS13, thus securing platelet tethering and thrombus adherence to the inflamed and injured endothelium, respectively (15). Furthermore, runt-related transcription factor 1 (RUNX1) plays a crucial role in the transition of endothelial cells to hematopoietic cells, and in the downregulation of fetal liver kinase-1. By contrast, Runx1 is weakly expressed in early erythroid cells, and its expression is rapidly extinguished during later stages of erythropoiesis $(16,17)$. In 


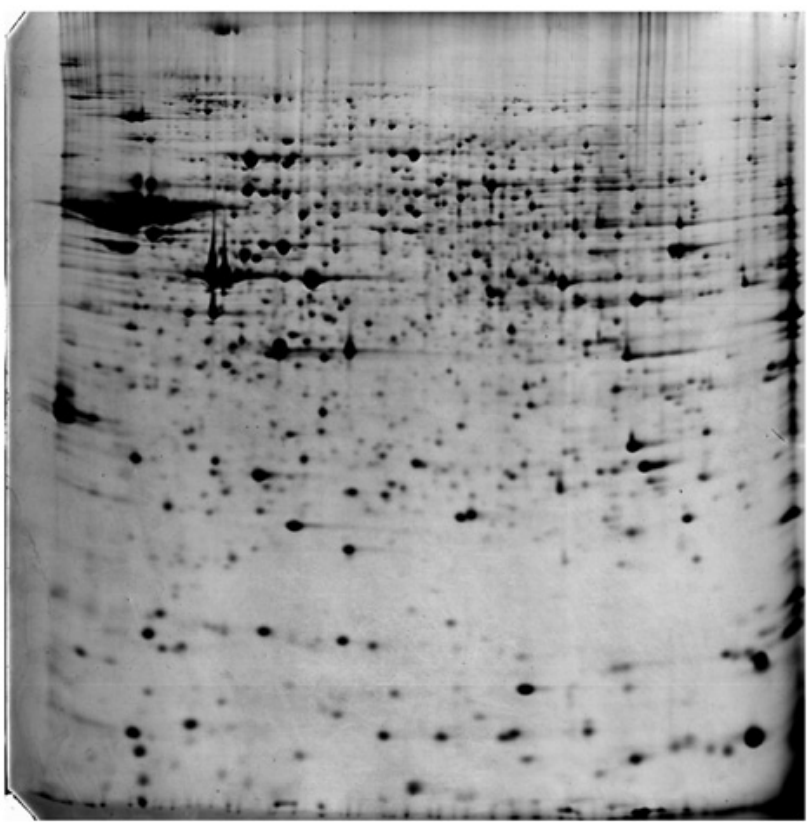

Figure 3. Two-dimensional polyacrylamide gel electrophoresis. Proteins from a lysate of pCMV-maspin-transfected LC5 cell line 3 were subjected to $18 \mathrm{~cm}$ pH3-10 (L) stripes by the GE Healthcare two-dimensional gel electrophoresis system. pCMV, porcine cytomegalovirus

addition, protein tyrosine kinase 2 (PTK2), or Focal adhesion kinase 1, plays a crucial role in generating cell survival signals, as well as the cleavage of FAK during caspase-mediated apoptosis (18). FAK catalytic activities are crucial in promoting VEGF-associated tumor angiogenesis and protease-associated tumor metastasis. Support is growing for the theory that FAK and Src may be therapeutically relevant targets in the inhibition of tumor progression (19).

\section{Discussion}

The findings of this study illustrate the importance of the MASPIN gene and protein in regulating the signaling pathway of tumor initiation, promotion and progression, which includes a very complicated and intricate control system with multiple layers that are connected to the expression of the MASPIN gene, including transcription factors, transcription factor binding elements and DNA methylation coalescence.

The expression level of MASPIN was used as an indicator for tumor aggressiveness and metastatic potential. Breast cancer progression from ductal carcinoma in situ, to locally invasive cancer, and finally to lymph node metastasis has been shown to correlate with a stepwise decrease in MASPIN expression (20). Umekita et al reported that the expression of MASPIN predicted poor prognosis in breast cancer patients (21). The down-regulation of the tumor suppressor gene, MASPIN, in breast carcinoma was associated with a higher risk of distant metastasis (20). In this study, we also demonstrate that MASPIN may be an indicator of aggressiveness and metastatic potential for lung cancer cells owing to its dual role in the inhibition of tumor cells.

Previous studies on the regulatory mechanisms of MASPIN have shown that hypermethylation and histone deacetylation lead to silencing of the MASPIN gene in human breast cancer $(22,23)$. Bass et al demonstrated that MASPIN was a non-inhibitory Serpin, which inhibited the migration of tumor and vascular smooth muscle cells (24). Recombinant MASPIN specifically inhibited activators of the cell surface-associated urokinase-type plasminogen and fibrinogen-bound tissue-type plasminogen. Endogenous MASPIN was also a potent inhibitor of the pericellular urokinase-type plasminogen activator and may block tumor invasion and motility by inhibiting localized pericellular proteolysis (25).

Jiang et al reported that MASPIN sensitizes breast carcinoma cells to induced apoptosis (26). Systemic delivery of the MASPIN gene in a syngeneic tumor model inhibited breast tumor progression (27). In this study, comparative proteomic analysis was used to systemically study the protein profile change in a lung cell line.

This study may help to illustrate the function of MASPIN, as well as the regulatory pathway and functional network of MASPIN in lung cancer. However, further studies are required to investigate the functions of MASPIN in further detail.

\section{References}

1. Zou Z, Gao C, Nagaich AK, et al: p53 regulates the expression of the tumor suppressor gene maspin. J Biol Chem 275: 6051-6054, 2000.

2. Shi HY, Zhang W, Liang R, et al: Modeling human breast cancer metastasis in mice: maspin as a paradigm. Histol Histopathol 18: 201-206, 2003.

3. Abraham S, Zhang W, Greenberg $\mathrm{N}$ and Zhang $\mathrm{M}$ : Maspin functions as tumor suppressor by increasing cell adhesion to extracellular matrix in prostate tumor cells. J Urol 169: 1157-1161, 2003.

4. Machtens S, Serth J, Bokemeyer C, et al: Expression of the p53 and Maspin protein in primary prostate cancer: correlation with clinical features. Int J Cancer 95: 337-342, 2001.

5. Schaefer JS and Zhang M: Role of maspin in tumor metastasis and angiogenesis. Curr Mol Med 3: 653-658, 2003.

6. Cher ML, Biliran HR Jr, Bhagat S, et al: Maspin expression inhibits osteolysis, tumor growth, and angiogenesis in a model of prostate cancer bone metastasis. Proc Natl Acad Sci USA 100: 7847-7852, 2003

7. Dokras A, Coffin J, Field L, et al. Epigenetic regulation of maspin expression in the human placenta. Mol Hum Reprod. 12: 611-617, 2006.

8. Brandt DT, Baarlink C, Kitzing TM, et al: SCAI acts as a suppressor of cancer cell invasion through the transcriptional control of beta1-integrin. Nat Cell Biol 11: 557-568, 2009.

9. Neznanov N, Neznanova L, Angres B and Gudkov AV: Serologically defined colon cancer antigen 3 is necessary for the presentation of TNF receptor 1 on cell surface. DNA Cell Biol 24: 777-785, 2005.

10. Nagasaki K, Schem C, von Kaisenberg C, et al: Leucine-zipper protein, LDOC1, inhibits NF-kappaB activation and sensitizes pancreatic cancer cells to apoptosis. Int J Cancer 105: 454-458, 2003.

11. Cicek M, Fukuyama R, Welch DR, Sizemore N and Casey G: Breast cancer metastasis suppressor 1 inhibits gene expression by targeting nuclear factor-kappaB activity. Cancer Res 65: 3586-3595, 2005.

12. Park S, Lim Y, Lee D, et al: Identification and characterization of a novel cancer/testis antigen gene CAGE-1. Biochim Biophys Acta 1625: 173-182, 2003.

13. Kim M, Gans JD, Nogueira C, et al: Comparative oncogenomics identifies NEDD9 as a melanoma metastasis gene. Cell 125: 1269-1281, 2006

14. Merrill RA, See AW, Wertheim ML and Clagett-Dame M: Crk-associated substrate (Cas) family member, NEDD9, is regulated in human neuroblastoma cells and in the embryonic hindbrain by all-trans retinoic acid. Dev Dyn 231: 564-575, 2004.

15. Bonnefoy A, Daenens K, Feys HB, et al: Thrombospondin-1 controls vascular platelet recruitment and thrombus adherence in mice by protecting (sub)endothelial VWF from cleavage by ADAMTS13. Blood 107: 955-964, 2006. 
16. Lorsbach RB, Moore J, Ang SO, Sun W, Lenny N and Downing JR: Role of RUNX1 in adult hematopoiesis: analysis of RUNX1-IRES-GFP knock-in mice reveals differential lineage expression. Blood 103: 2522-2529, 2004.

17. Hirai H, Samokhvalov IM, Fujimoto T, Nishikawa S, Imanishi J and Nishikawa S: Involvement of Runx1 in the down-regulation of fetal liver kinase-1 expression during transition of endothelial cells to hematopoietic cells. Blood 106: 1948-1955, 2005.

18. Schlaepfer DD, Hauck CR and Sieg DJ: Signaling through focal adhesion kinase. Prog Biophys Mol Biol 71: 435-478, 1999.

19. Bernard-Trifilo JA, Lim ST, Hou S, Schlaepfer DD and Ilic D: Analyzing FAK and Pyk2 in early integrin signaling events. Curr Protoc Cell Biol Chapter 14: Unit 14.7, 2006.

20. Maass N, Teffner M, Rosel F, et al: Decline in the expression of the serine proteinase inhibitor maspin is associated with tumour progression in ductal carcinomas of the breast. J Pathol 195: 321-326, 2001.

21. Umekita Y, Ohi Y, Sagara Y and Yoshida H: Expression of maspin predicts poor prognosis in breast-cancer patients. Int $\mathrm{J}$ Cancer 100: 452-455, 2002.
22. Futscher BW, Oshiro MM, Wozniak RJ, et al: Role for DNA methylation in the control of cell type specific maspin expression. Nat Genet 31: 175-179, 2002.

23. Maass N, Biallek M, Rosel F, et al: Hypermethylation and histone deacetylation lead to silencing of the maspin gene in human breast cancer. Biochem Biophys Res Commun 297: 125-128, 2002.

24. Bass R, Fernandez AM and Ellis V: Maspin inhibits cell migration in the absence of protease inhibitory activity. J Biol Chem 277: 46845-46848, 2002.

25. Biliran H Jr and Sheng S: Pleiotrophic inhibition of pericellular urokinase-type plasminogen activator system by endogenous tumor suppressive maspin. Cancer Res 61: 8676-8682, 2001.

26. Jiang N, Meng Y, Zhang S, Mensah-Osman E and Sheng S: Maspin sensitizes breast carcinoma cells to induced apoptosis. Oncogene 21: 4089-4098, 2002.

27. Shi HY, Liang R, Templeton NS and Zhang M: Inhibition of breast tumor progression by systemic delivery of the maspin gene in a syngeneic tumor model. Mol Ther 5: 755-761, 2002. 